\title{
Building up a Continuous Flow Platform as an Enabler to the Preparation of Intermediates on Kilogram Scale
}

\author{
Edouard Godineau, ${ }^{\text {,a }}$ Claudio Battilocchio, ${ }^{\text {*a }}$ and Mukul Lal ${ }^{\mathrm{b}}$
}

\begin{abstract}
The following outlook describes the strategy we followed at Syngenta R\&D to build and develop an effective flow chemistry platform which could fit a precise business purpose. In this account, we give insight into specific chemistry challenges encountered and addressed using continuous flow chemistry. The conclusions of the outlook outline the future of our strategy with a perspective on the technology within our business.
\end{abstract}

Keywords: Bromination · Continuous flow chemistry · Hazardous chemistry · Nitration · Scale-up

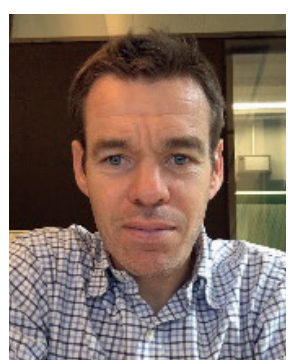

Edouard Godineau received his $\mathrm{PhD}$ from the University of Bordeaux in 2007, with Prof. Yannick Landais. After a postdoctoral stay with Prof. A- Fürstner, he joined Syngenta Crop Protection in 2009 as a process chemist. From 2014 to 2019 , he was leading the process chemistry group at Syngenta and was responsible to identify and design most cost-effective possible synthetic routes to new active ingredients. Since February 2019, he holds the position of senior scientific expert in process chemistry at Syngenta.

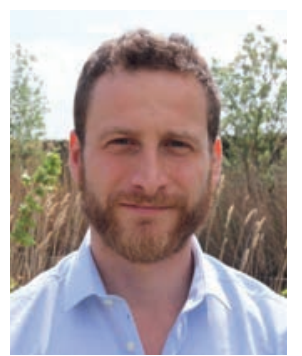

Claudio Battilocchio received his $\mathrm{PhD}$ from La Sapienza University of Rome in 2012, with the title of Doctor Europeaus, spending half of his studies at the University of Cambridge. He was a postdoc in the group of Prof. Steven V. Ley (University of Cambridge), working on various applications of Flow chemistry technologies with Pfizer (2012-2015), and then with Syngenta (2015-2017). Claudio joined Syngenta at the end of 2017, where he currently leads the flow chemistry research platform.

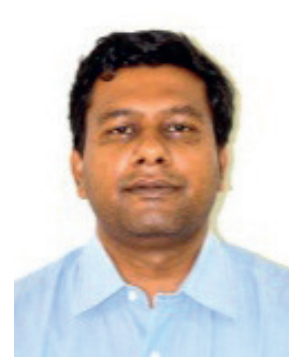

Mukul Lal received his $\mathrm{PhD}$ from the University of Siegen in 2005, with Prof. Michael Schmittel and a post-doctoral work with Prof. Martin Newcomb at University of Illinois at Chicago. He started his industrial career with GVK-Bio as senior research scientist in Medicinal Chemistry. In 2007 he moved to BASF India Ltd. as Team Lead in performance chemicals. In 2010 he joined the kilo lab platform of Syngenta in Goa and since 2013 he is leading the platform.

\section{Introduction}

Within the last decade, at Syngenta R\&D, we have seen an increasing demand for larger and larger amounts of intermediates and final products at earlier stages of the development phases. In a general workflow, hundreds of grams of such intermediates/ products are generally required when neither process evaluation nor process design have been initiated; therefore, a rather 'crude' discovery route might be the only solution available. While in a large number of cases the traditional batch approach suits the purpose for scaling-up, in other cases this is not ideal and, even worse, can present challenges sometimes difficult to address. Some of the challenges we refer to are safety issues (e.g. a reaction which generates an unstable and hazardous intermediate difficult to handle in batch), others might be operational issues (e.g. high temperature reactions, use of gaseous reagents), or challenges depending on the chemistry itself (e.g. selectivity).

Continuous flow chemistry represents an efficient solution to many processing problems. Continuous flow chemistry is certainly not a universal solution but becomes a powerful tool to address well-identified issues. ${ }^{[1]}$ In this outlook article, we would like to highlight how we have been using the attributes of this technology to timely access larger amounts of material needed, in a simpler, safer and more effective way. Our aim in this article is not to discuss in depth the technical abilities of continuous flow technology (e.g. providing better mixing, heat dissipation and/or control on selectivity) but rather to describe the strategy we followed at Syngenta R\&D: a strategy to build and develop an effective internal platform. We are hoping to demonstrate with a selection of relevant examples of how we have achieved some of our goals and delivered challenging internal demands for material.

\section{Time-demanding Operations}

The development of new active ingredients in the agrochemical sector underwent a series of steep changes to address regulatory challenges. This means that R\&D of new active ingredients would commonly include, at early stages, thorough toxicological evaluations in addition to clearly demonstrating high selectivity towards the targeted pest. As a consequence, larger amounts of material are needed at these early stages of research programs. In general, the first synthesis designed by the discovery chemists is meant to achieve one objective: the rapid delivery of material for early biological evaluation. When a successful hit is identified, additional material is quickly required. At this stage, the attrition rate is still relatively high and time is the most critical factor. In 
most cases, no time is available for the chemist to optimize or to redesign the original discovery route in order to simplify the production of material in large quantities.

\section{KiloLab and Scale-up Challenges}

Our KiloLab facility, located in Goa (India), routinely uses such 'discovery routes' to produce material on a $500 \mathrm{~g}$ to $1000 \mathrm{~g}$ scale. Our facilities are equipped with batch reactors of various sizes (up to $100 \mathrm{~L}$ ) and most standard purification systems. Our KiloLab effectively delivers intermediate and product requests from the entire Syngenta R\&D function. However, significant hurdles are sometimes encountered. Safety often presents as a primary challenge. For example, fairly large amounts $(>1 \mathrm{Kg})$ of nitropyridine $\mathbf{3}$ were required for a campaign. For this purpose, our strategy was to follow the literature procedure whereby ${ }^{[2]}$ nitration of 2-trifluoromethylpyridine-N-oxide 2, (Scheme 1a) delivers the title compound upon work-up. Preliminary analysis of scale-up operations highlighted technical challenges since the reaction conditions required treatment with $\mathrm{H}_{2} \mathrm{SO}_{4} / \mathrm{HNO}_{3}$ at high temperatures $\left(85-125^{\circ} \mathrm{C}\right)$. Reaction control measures such as dosing of substrate under the reaction conditions proved to be operationally challenging at high temperatures. In addition, thorough safety investigations indicated clear risks, which needed careful assessment. After thorough risk analysis, it was decided that the entire amount of material be delivered using multiple reaction batches, with a maximum scale of $c a .100 \mathrm{~g}$ for each batch reaction.

Similarly, another example, where strong safety issues were identified, involved a cycloaddition process using dibromoformaldoxime $\mathbf{4}$, as a reactive intermediate (Scheme $1 \mathrm{~b}$ ). In this particular case, we rapidly realized that $\mathbf{4}$, although commercially available, was an unsafe intermediate to produce, isolate and react at scale. ${ }^{[3]}$ In order to manage the safety risks appropriately, we had to limit the operations of scale-up to repetitions of smaller size batches, in order to deliver the quantities needed.

Another classical problem is reproducibility upon scaling up. We met such an example in the bromination of methyl ketone 8 (Scheme 1c), where our experiments resulted in very variable yields depending on the reaction scale, demonstrating a clear lack of reproducibility. Although on small scale we could control relatively well the selectivity of the reaction, on a $150 \mathrm{~g}$ scale much lower yields (average 30\%) of the target ketone 9 were obtained, with larger amounts of the di-brominated ketone $\mathbf{1 0}$ being detected. Such observations depend on selectivity issues, which are very well understood, ${ }^{[4]}$ and can be attributed to the 'non-ideal' mixing scenario which worsens upon scale-up. Once again, we limited our reaction scale to small batches, in order to reliably achieve rapid delivery of material.

\section{Continuous Flow Chemistry as a Solution}

With increasing knowledge available on the technique in the last decade, we realized that continuous flow chemistry is a technology which offers particular advantages to overcome the aforementioned issues. In particular, we were very much interested in the technology for the following benefits: ${ }^{[1]}$

- Excellent control and mitigation of the risk in handling hazardous reagents, as during the whole process, the amounts of reactants and reagents present within the reactor are very small compared to the corresponding batch scenario and are contained; ${ }^{[1]}$

- Heat transfer is significantly improved, enabling better handling of the scale-up of highly exothermic reactions; $[1 \mathrm{~b}, \mathrm{~g}]$

- Extremely fast reactions are better controlled (yield, selectivity, safety) because of control on mixing phenomena; $[1 \mathrm{k}]$

- Telescoping of reaction sequences can be achieved, such that unstable intermediates are generated and consumed 'on-demand', without need for isolation; $[1 \mathrm{a}, \mathrm{k}]$

- Reaction conditions are carefully controlled thanks to the fine adjustment of residence/reaction times.

\section{Flow Technology to Simplify Scale-up in Selected Cases}

We anticipated that the development of an internal continuous flow chemistry platform would result in clear benefits and directly impact on our daily needs. Not only would this bring a complementary approach to established batch techniques but also boost our synthesis efficiency in selected cases.

We started in 2014 at Syngenta building the first continuous flow chemistry capabilities for research purposes. Acquiring equipment, engaging with local engineering support, building know-how via our network with neighboring industries and academic partners were key factors enabling the implementation of the technology in our site in Stein (Switzerland). An example of fruitful knowledge transfer process was established within a collaborative scheme with Prof. Steven Ley. The aim for the collaboration was to significantly accelerate the development of our platform and boost our knowhow in the field so as to be fit to tackle a targeted range of problematic reactions. Together with the Ley group, we were able to bring an answer to the scale-up issue caused by the above-mentioned handling of dibromoformaldoxime $4{ }^{[3]} \mathrm{A}$ fully integrated approach was designed at the University of Cambridge, UK (Scheme 2), whereby dibromoformaldoxime $\mathbf{4}$ is generated on-demand and immediately consumed, with no accumulation of this hazardous intermediate, enabling a smooth and safe scale-up. As part of the collaboration's objective, the multistep process was replicated in our Stein laboratory. This transfer of know-how greatly contributed to the development of our internal platform.

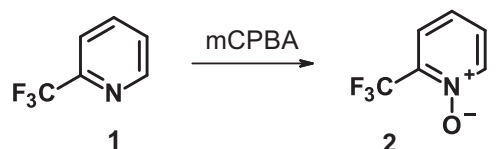

1
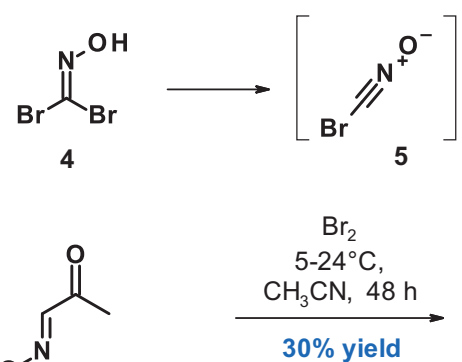

$30 \%$ yield b)

c)

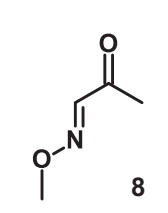

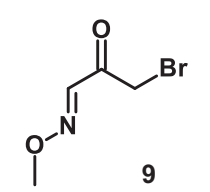

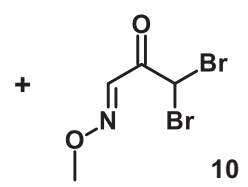




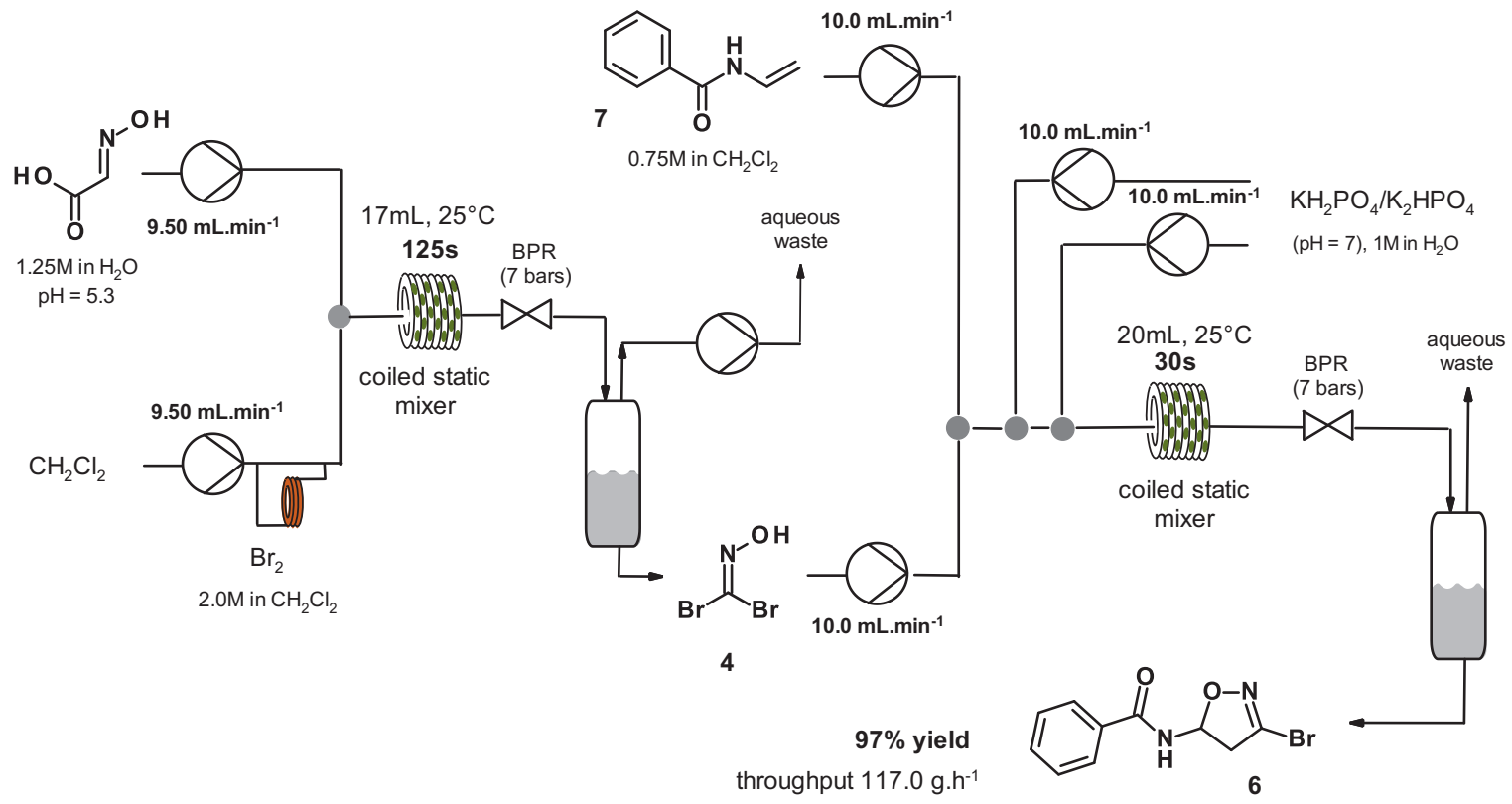

Scheme 2. Continuous flow set-up for the telescoped synthesis of isoxazoline 6.

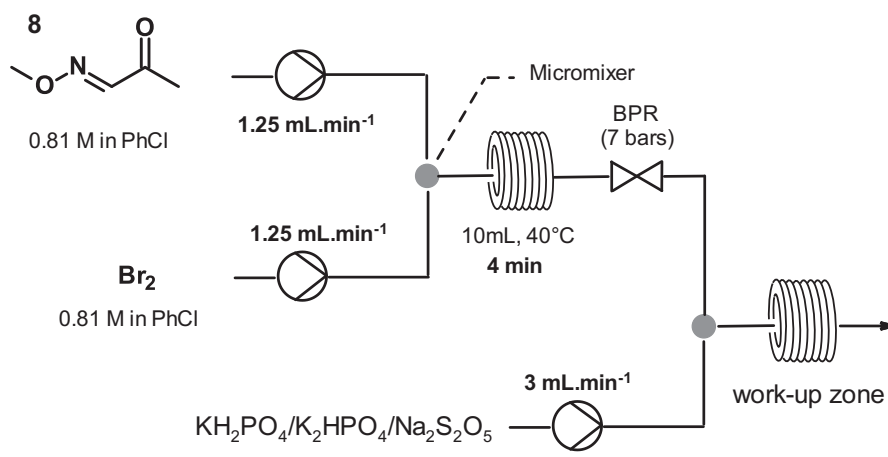

Scheme 3. Continuous flow bromination with in situ quench.
We quickly needed to access fairly large amounts of material. Thanks to some process intensification optimization, our research continuous flow set-up (which fitted in a standard hood) became fit for potentially delivering several hundreds of grams of material in a single working day. We demonstrated it, in Stein, by delivering ca. $200 \mathrm{~g}$ of the isoxazoline intermediate $\mathbf{6}$ without any noticeable issues.

Similarly, we could also design a solution to the problematic bromination briefly described earlier. In this instance, the target bromoketone 9 could be produced in high yield and high selectivity thanks to the improved mixing and improved heat control of our designed continuous process (Scheme 3). ${ }^{[5]}$ Our scale-up process clearly indicated the protocol was highly reproducible and robust over time.

A general class of reaction for which continuous flow offers great advantages is Li-halogen exchange type chemistry, followed by quenching with an electrophile. Such reactions are indeed known to be very well-suited for the technology since they are extremely fast and highly exothermic. ${ }^{[1 b, 6]}$ In batch mode, such reactions are typically run under cryogenic conditions. In continuous mode - thanks to excellent heat dissipation properties - both the Li-halogen exchange and quenching steps can be performed at room temperature (or generally speaking, temperatures closer to $20-25^{\circ} \mathrm{C}$ ). This is a significant benefit on larger scale (time and energy savings). An additional value is the better control of selectivity and by-product formation, which usually results in direct observable impact on yield and reproducibility. The example shown in Scheme 4 showcases such advantages appropriately. In batch mode, despite very careful reaction control, variable yields of the desired alkoxide $\mathbf{1 3}$ (from 50\% up to 74\%) were obtained. In sharp contrast, the alternative continuous set- up we designed delivered the product in $95 \%$ yield, in a very reproducible way.

Again, larger amounts of alkoxide $\mathbf{1 3}$ were required. We managed to intensify our continuous process such that our set-up (hood) was able to deliver several hundreds of grams of material in a single working day. However this time, we anticipated that our Swiss research labs would no longer be fit to handle the fairly large amounts of crude reaction mixture continuously produced. We foresaw as well significant pragmatic difficulties in performing, in our research labs, downstream processing operations on greater than $\mathrm{kg}$ scale in order to isolate our intermediate $\mathbf{1 3}$.

We solved these issues by replicating our continuous process within our KiloLab facility in India. Over there, the accumulation and the processing of the large volume of crude reaction mixture (few liters) were no longer problematic. To our delight, no significant differences (yield, output quality) were observed, and our KiloLab team was able to deliver $4 \mathrm{~kg}$ of the target alcohol, without any noticeable issues.

Our journey to establishing our continuous flow platform in R\&D was exciting and today we see the technology as an accessible alternative for scaling-up chemistry, when tangible benefits are foreseen. Technical expertise about pros and cons of the technology is a key asset of our team and is essential know-how, to spot, select and prioritize opportunities to tackle. This ensures we use the technology "whenever it makes sense" and helps maximizing the impact of the team.

\section{Present and Future Meet our Ambition}

Our platform has proven to be relatively successful so far. Within the last two years, we were able to demonstrate the advantages of 

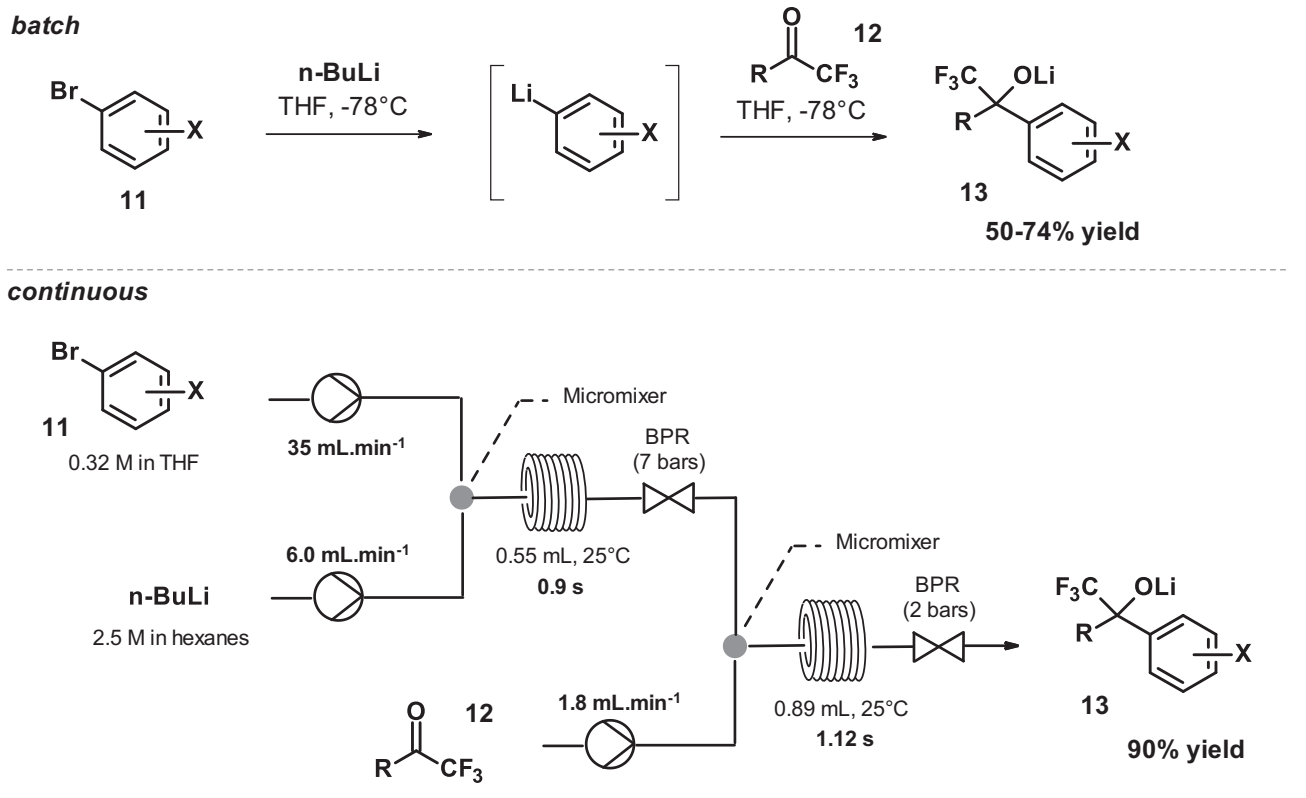

$7.05 \mathrm{M}$ (neat)

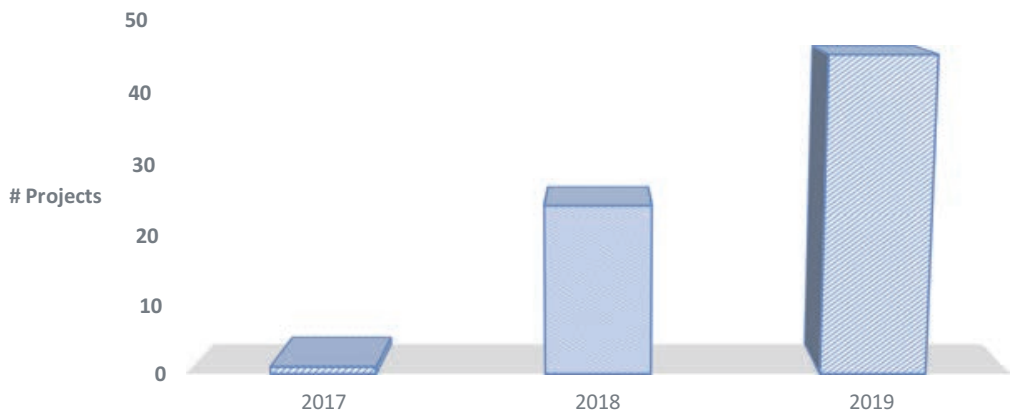

Scheme 4. Continuous flow lithiation and quench with electrophiles to yield the fluorinated alkoxide 13.

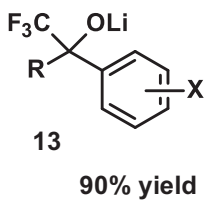

Fig. 1. Number of internal projects selected as potential opportunities to explore with continuous flow chemistry. the technology. This is clearly highlighted by the rapid growing number of 'opportunities' addressed in different projects (Fig. 1).

We have the ambition, in upcoming years, to perform reactions (e.g. the lithium-halogen example above-mentioned) which show clear benefits, only in continuous mode. We are also hoping to extend our portfolio of such examples to other classes of reactions (e.g. nitrations $\left.{ }^{[7]}\right)$. Since the early stages of implementation, we have efficiently designed in our research laboratory in Stein processes which showed tangible benefits (over the classical batch mode) for accessing larger amounts of material. We can enumerate several transformations to date, such as Curtius rearrangement, triazole synthesis, electrophilic chlorination and bromination, high temperature decarboxylation, and several low/ high temperature reactions.

Although batch mode still holds a premium place in the chemist's toolbox, we anticipate the technology will, in the coming years, be regarded by the chemist as a routine and well accessible alternative to prepare compounds. We believe the future of Continuous Flow Chemistry will be focused on four major pillars:

1. Safer and sustainable chemistry. Our goal for the future will be to embrace more and more automation from early stages of discovery, using continuous flow when a clear advantage is identified. This will certainly help shaping our continuous flow chemistry 2.0 platform.

2. More efficiency and productivity. The use of AI and software control will relieve chemists of routine tasks and enable a more productive, more efficient working model. Moreover, the use of AI will certainly address the necessity of exploring the entire 3D chemical space.

3. Streamlining data and learnings. Data modelling and ability to predict reaction scalability based on engineering parame- ters, will be a fundamental addition to our platform tools - this will drive future applications.

4. Educating people. People know-how is at the core of the discipline and will be a pivotal aspect to affect decision making processes.

Received: September 18, 2019

[1] a) J. C. Pastre, D. L. Browne, S. V. Ley, Chem. Soc. Rev. 2013, 42, 8849; b) J. A. Newby, D. W. Blaylock, P. M. Witt, J. C. Pastre, M. K. Zacharova, S. V. Ley, D. L. Browne, Org. Proc. Res. Dev. 2014, 18, 1211; c) M. Movsisyan, E. I. P. Delbeke, J. K. E. T. Berton, C. Battilocchio, S. V. Ley, C. V. Stevens, Chem. Soc. Rev. 2016, 45, 4892; d) N. Kockmann, M. Gottsponer, B. Zimmermann, D. M. Roberge, Chem. - Eur. J. 2008, 14, 7470; e) J. Hartwig, J. B. Metternich, N. Nikbin, A. Kirschning, S. V. Ley, Org. Biomol. Chem. 2014, 12, 3611; f) M. Baumann, I. R. Baxendale, Beilstein J. Org. Chem. 2015, 11, 1194; g) T. Razzaq, C. O. Kappe, Chem. - Asian J. 2010, 5, 12741289; h) M. Irfan, T. N. Glasnov, C. O. Kappe, ChemSusChem 2011, 4, 300; i) B. Gutmann, D. Cantillo, C. O. Kappe, Angew. Chem. Int. Ed. 2015, 54, 6688; j) S. V. Ley, I. R. Baxendale, Chimia 2008, 62, 3, 162; k) P. Plouffe, A. Macchi, D. M. Roberge, Org. Proc. Res. Dvp. 2014, 18, 11, 1286; 1) R. J. Ingham, C. Battilocchio, D. E. Fitzpatrick, E. Sliwinski, J. M. Hawkins, S. V. Ley, Angew. Chem. Int. Ed. 2015, 54, 144.

[2] a) Y. Oguro, N. Miyamoto, T. Takagi, K. Okada, Y. Awazu, H. Miki, A Hori, K. Kamiyama, S. Imamura, Bioorg. Med. Chem. 2010, 18, 7150; b) B. Aquila, P. Lyne, T. Pontz, WO2007113558A2, 2007.

[3] C. Battilocchio, F. Bosica, S. M. Rowe, B. L. Abreu, E. Godineau, M. Lehmann, S. V. Ley, Org. Proc. Res. Dev. 2017, 21, 1588.

[4] a) J. R. Bourne, P. Rys, K. Suter, Chem. Eng. Sci. 1977, 32, 711; b) J. R. Bourne, Org. Proc. Res. Dev. 2003, 7, 471.

[5] E. Godineau, C. Battilocchio, M. Lehmann, S. V. Ley, R. Labes, L. Birnoschi, S. Subramanian, C. S. Prasanna, A. Gorde, M. Kalbagh, V. Khade, A. Scherrer, A. C. O'Sullivan, Org. Proc. Res. Dev. 2018, 22, 8, 955.

[6] A. Hafner, P. Filipponi, L. Piccioni, M. Meisenbach, B. Schenkel, F. Venturoni, J. Sedelmeier, Org. Proc. Res. Dev. 2016, 20, 1833.

[7] A. A. Kulkarni, Beilstein J. Org. Chem. 2014, 10, 405. 